\title{
Accelerated adiabatic quantum gates: Optimizing speed versus robustness
}

\author{
Hugo Ribeiro ${ }^{1, *}$ and Aashish A. Clerk ${ }^{2}$ \\ ${ }^{1}$ Max Planck Institute for the Science of Light, Staudtstraße 2, 91058 Erlangen, Germany \\ ${ }^{2}$ Institute for Molecular Engineering, University of Chicago, 5640 South Ellis Avenue, Chicago, Illinois 60637, USA
}

(Received 25 June 2019; published 17 September 2019)

\begin{abstract}
We develop protocols for high-fidelity single-qubit gates that exploit and extend theoretical ideas for accelerated adiabatic evolution. Our protocols are compatible with qubit architectures where direct transitions between logical states are either vanishingly small or nonexistent; in such systems traditional approaches cannot be implemented. Prime examples are superconducting fluxonium qubits, which have highly localized states, and AMO systems, where there are no dipole allowed transitions between the ground states encoding the logical states. By using an accelerated adiabatic protocol we can enforce the desired adiabatic evolution while having gate times that are comparable to the inverse adiabatic energy gap (a scale that is ultimately set by the amount of power used in the control pulses). By modeling the effects of decoherence, we explore the trade-off between speed and robustness that is inherent to shortcuts-to-adiabaticity approaches.
\end{abstract}

DOI: 10.1103/PhysRevA.100.032323

\section{INTRODUCTION}

Any approach to implementing quantum gates requires applying time-dependent control fields to a system, such that the corresponding time-dependent Hamiltonian generates the desired unitary evolution. Regardless of the setting, ideal gates have two defining features: they are both robust against small imperfections in the amplitude, duration, and phase of control pulses, and they are fast. Unfortunately, typical approaches to constructing gates optimize only one of these two desired characteristics. Schemes based on quantum adiabatic evolution (e.g., [1,2]) are typically extremely robust against parameter variations [3] but suffer from extremely long protocol times. In contrast, more conventional nonadiabatic approaches can be extremely fast (approaching the quantum speed limit [4-9]) but require precise tuning of control pulses. In a typical experimental setting, neither approach is fully optimal, as both speed and robustness are important characteristics.

Given this, protocols that lie between these two extremes are highly desirable. This naturally leads one to the general approach of shortcuts to adiabaticity (STAs) [10-13] (also known as counter-diabatic driving). STAs are a family of techniques that allow one to mimic adiabatic evolution under some Hamiltonian $\hat{H}_{0}(t)$ using a modified Hamiltonian $\hat{H}_{\text {mod }}(t)$, on a much shorter time scale. STA protocols for evolving a well-defined initial state to some prescribed well-defined final state have been discussed in many contexts and have even recently been implemented in a variety of experimental settings [14-20]. While not often stressed, STA protocols invariably involve a tunable trade-off between speed and robustness. This tunability can, however, be extremely useful in a real experimental setting, where the ultimate infidelity of a gate will be influenced by both these features. In Ref. [19], this trade-off was discussed in the specific context of an

\footnotetext{
*hugo.ribeiro@mpl.mpg.de
}

accelerated STIRAP protocol implemented in an NV center system.

In this paper, we investigate the use of STA techniques to accelerate well-known adiabatic quantum gates based on a tripod level configuration, where three "ground-state" levels all interact controllably with a single "excited-state" level. Such schemes can find direct application in a variety of systems, including trapped ion qubits $[1,21]$ as well as superconducting qubits $[22,23]$. Accelerating a quantum gate is a more challenging problem than simply accelerating an adiabatic evolution with a single, well-defined initial state, as now one is interested in a manifold of possible initial states. In the case of a unique initial state, the (global) phase accumulated during the evolution is of no importance and it can consequently differ between the adiabatic and the accelerated protocols. In stark contrast, when generating a quantum gate, the accumulated phases are of utmost importance. This is problematic, as standard STA techniques are not designed to preserve dynamical or geometric phases generated by adiabatic evolution. Despite this difference, we show that the superadiabatic transitionless driving (SATD) scheme developed in Ref. [24] to accelerate STIRAP-style quantum state transfer can be used to accelerate tripod-based adiabatic quantum gates. We also study in detail the trade-offs entailed when using an accelerated protocol: while the protocol time can be dramatically reduced, one necessarily also becomes less tolerant of parameter variations and more sensitive to dissipative effects originating with the lossy excited level. The understanding we develop will allow one to design an optimally constructed accelerated protocol for a given set of experimental parameters.

In contrast to other schemes [25-28] that generate nonadiabatic geometric gates, ours is purely geometric and does not rely on accumulating specific dynamical phases. This also distinguishes our work from the recent experiment by Yan et al. [29], where an accelerated geometric gate is only obtained if a dynamical phase is canceled by applying a $\pi$ pulse. We also note that while Ref. [30] straightforwardly 
(a)

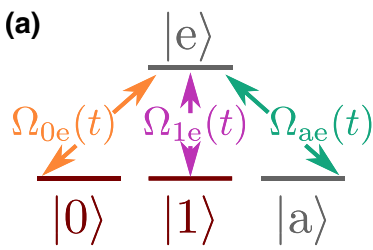

(c)

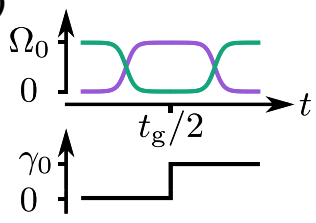

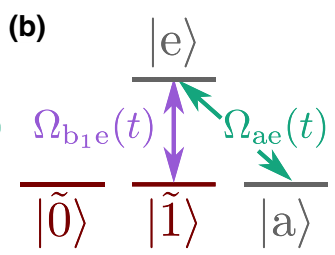

(d)

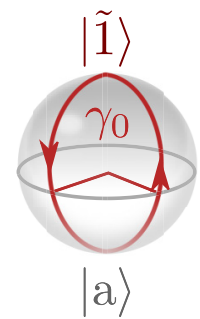

FIG. 1. (a) Schematic of a tripod system. We choose $|0\rangle$ and $|1\rangle$ to encode the qubit states. The ground-state manifold couples to an excited state $|e\rangle$ via control fields denoted $\Omega_{0 e}(t), \Omega_{1 e}(t)$, and $\Omega_{a e}(t)$. (b) Effective $\Lambda$ system describing the evolution of the tripod system. The control pulses $\Omega_{b_{1} e}(t)$ and $\Omega_{a e}(t)$ are chosen to generate a cyclic evolution based on STIRAP. (c) Example of a control sequence generating a cyclic evolution. The relative phase of $\Omega_{a e}(t)$ is changed instantaneously at $t=t_{g} / 2$. This does not result in any discontinuity in the evolution since $\Omega_{a e}\left(t_{g} / 2\right)=0$. (d) Geometric representation of the evolution of the system on the Bloch sphere. The geometric phase accumulated by $|\tilde{1}\rangle$ is equal to the solid angle encapsulated during the evolution.

applied the dressed-state technique of Ref. [24] to accelerate an adiabatic gate, the authors did not consider the potential difficulties associated with this procedure (stemming from STA-induced modification of phases).

\section{GEOMETRIC GATES IN A TRIPOD SYSTEM}

\section{A. Basic double-STIRAP protocol}

We start by reviewing how geometric qubit gates can be implemented in a four-level tripod system [see Fig. 1(a)]. Our discussion complements existing literature $[1,2]$ by providing a thorough discussion of how nonadiabatic errors deteriorate the performance of such gates. The system consists of three ground-state levels, $(|0\rangle,|1\rangle$, and $|a\rangle)$, each of which is controllably and resonantly coupled to a common excited state $|e\rangle$. The system Hamiltonian (in the rotating frame of the drive fields) is

$$
\begin{aligned}
\hat{H}(t)= & \frac{1}{2}\left[\Omega_{0 e}(t)|0\rangle\left\langle e\left|+\Omega_{1 e}(t)\right| 1\right\rangle\langle e|\right. \\
& \left.+\Omega_{a e}(t)|a\rangle\langle e|+\text { H.c. }\right],
\end{aligned}
$$

where $\Omega_{i e}(t)(i=0,1, a)$ denotes the complex envelope of each control field.

We use the ground states $|0\rangle$ and $|1\rangle$ to encode a logical qubit state (logical subspace). It allows one to use highly isolated states as qubit levels, thus potentially enabling long coherence times. This kind of situation can be realized in a variety of experimental platforms, e.g., in superconducting fluxonium qubits [22,23].

We next parametrize the control fields, assuming only that they are chosen to keep the instantaneous eigenvalues of $\hat{H}(t)$

independent of time:

$$
\begin{aligned}
& \Omega_{0 e}(t)=\Omega_{0} \cos (\alpha) \sin [\theta(t)], \\
& \Omega_{1 e}(t)=\Omega_{0} \sin (\alpha) \sin [\theta(t)] e^{i \beta}, \\
& \Omega_{a e}(t)=\Omega_{0} \cos [\theta(t)] e^{i \gamma(t)} .
\end{aligned}
$$

$\Omega_{0}$ determines the overall scale for the control fields [and the size of the energy gap of $\hat{H}(t)]$, while the angles $\theta$ and $\alpha$ determine their relative magnitudes. The angles $\beta$ and $\gamma$ control the relative phases between control fields. For reasons that will become clear, we consider in what follows protocols where $\alpha$ and $\beta$ are time independent. We emphasize that choosing $\Omega_{0}$ to be constant in Eq. (2) is not restrictive. As we discuss below, adding a finite turn-on (turn-off) to the control pulses does not modify the dynamics of the logical subspace.

Diagonalizing the instantaneous Hamiltonian $\hat{H}(t)$, one finds that it always possesses two zero-energy eigenstates that are orthogonal to $|e\rangle$. States in this "dark-state" manifold are ideally suited for geometric gates, as they will never acquire dynamical phases. Further, there is always a unique dark state that is a superposition of qubit states only, namely,

$$
|\tilde{0}\rangle=\sin (\alpha)|0\rangle-\exp (i \beta) \cos (\alpha)|1\rangle .
$$

This state does not depend on time. The orthogonal qubit-only state is

$$
|\tilde{1}\rangle=\cos (\alpha)|0\rangle+\exp (i \beta) \sin (\alpha)|1\rangle
$$

and, in general, is not an instantaneous eigenstate of $\hat{H}(t)$.

Writing $\hat{H}(t)$ in terms of these new qubit basis states yields

$$
\hat{H}(t)=\frac{1}{2}\left[\Omega_{\tilde{1} e}(t)|\tilde{1}\rangle\left\langle e\left|+\Omega_{a e}(t)\right| a\right\rangle\langle e|+\text { H.c. }\right],
$$

where $\Omega_{\tilde{1} e}(t)=\Omega_{0} \sin [\theta(t)]$. We see that the qubit state $|\tilde{0}\rangle$ is completely decoupled, whereas the qubit state $|\tilde{1}\rangle$ forms a three-level $\Lambda$ system [31,32] with the states $|a\rangle$ and $|e\rangle$ [see Fig. 1(b)]. One can now use well-known STIRAP protocols [31,32] to adiabatically manipulate these states. In particular, using an appropriate double-STIRAP protocol we can engineer a cyclic evolution, such that the qubit state $|\tilde{1}\rangle$ acquires a purely geometric Berry phase [33,34]. This will form the basis of our adiabatic single-qubit gate (as first suggested in Refs. [1,2]).

To understand the double-STIRAP protocol, we first list the remaining instantaneous eigenstates of $\hat{H}(t)$. In addition to the zero-energy qubit dark state $|\tilde{0}\rangle$ [cf. Eq. (3)], $\hat{H}(t)$ in Eq. (5) also has a second, orthogonal zero-energy dark state,

$$
\left|d_{2}(t)\right\rangle=\cos [\theta(t)]|\tilde{1}\rangle-e^{i \gamma(t)} \sin [\theta(t)]|a\rangle,
$$

as well as two nonzero energy eigenstates,

$$
\left|b_{ \pm}(t)\right\rangle=\frac{1}{\sqrt{2}}\left( \pm \sin [\theta(t)]|\tilde{1}\rangle \pm e^{i \gamma(t)} \cos [\theta(t)]|a\rangle+|e\rangle\right)
$$

with instantaneous energies $\pm \Omega_{0} / 2$.

The double-STIRAP protocol involves adiabatically evolving the dark state $\left|d_{2}(t)\right\rangle$ from being purely $|\tilde{1}\rangle$ at $t=0$, to being $|a\rangle$ at $t=t_{g} / 2$, and then back to being $|\tilde{1}\rangle$ at the final time $t=t_{g}$. This can be accomplished by choosing

$$
\theta(t)= \begin{cases}\frac{\pi}{2} P(t), & 0 \leqslant t<\frac{t_{g}}{2}, \\ \frac{\pi}{2}\left[1-P\left(t-\frac{t_{g}}{2}\right)\right], & \frac{t_{g}}{2} \leqslant t \leqslant t_{g}\end{cases}
$$


where $P(t)$ is a monotonic function varying between $P(0)=0$ and $P\left(t_{g} / 2\right)=1$. The following form for $P(t)$ is particularly effective:

$$
P(t)=6\left(\frac{2 t}{t_{g}}\right)^{5}-15\left(\frac{2 t}{t_{g}}\right)^{4}+10\left(\frac{2 t}{t_{g}}\right)^{3}
$$

This choice gives a smooth turn-on and turn-off of the pulses, i.e., it satisfies $\dot{\theta}(0)=\dot{\theta}\left(t_{g} / 2\right)=\dot{\theta}\left(t_{g}\right)=\ddot{\theta}(0)=\ddot{\theta}\left(t_{g} / 2\right)=$ $\ddot{\theta}\left(t_{g}\right)=0$. Note that at this stage, we do not specify the time dependence of the relative phase $\gamma(t)$; as we will see, $\gamma(t)$ will determine the geometric phase acquired by $|\tilde{1}\rangle$. We assume for clarity that the control field $\Omega_{a e}(t)$ is nonzero at $t=0$. This is not restrictive. Even if one includes a finite turn-on (turn-off) time for this field, the additional resulting dynamics only affects the states $\{|a\rangle,|e\rangle\}$ (i.e., the auxiliary subspace). As shown in what follows, this does not hinder the realization of our geometric gate, as this gate is not contingent on any special preparation of the auxiliary subspace.

The system dynamics is best analyzed in the instantaneous eigenstate frame (adiabatic frame) that diagonalizes $\hat{H}(t)$ at each instant in time. The frame-change operator is given by

$$
\hat{S}_{\mathrm{ad}}(t)=|\tilde{0}\rangle\left\langle\tilde{0}|+| d_{2}(t)\right\rangle\left\langle d_{2}|+| b_{-}(t)\right\rangle\left\langle b_{-}|+| b_{+}(t)\right\rangle\left\langle b_{+}\right| .
$$

In the adiabatic frame, we have

$$
\begin{aligned}
\hat{H}_{\mathrm{ad}}(t) & =\hat{S}_{\mathrm{ad}}^{\dagger}(t) \hat{H}(t) \hat{S}_{\mathrm{ad}}(t)-i \hat{S}_{\mathrm{ad}}^{\dagger}(t) \partial_{t} \hat{S}_{\mathrm{ad}}(t) \\
& =\hat{H}_{0}(t)+\hat{V}_{\mathrm{err}}(t),
\end{aligned}
$$

where

$$
\begin{aligned}
\hat{H}_{0}(t)= & -\frac{\Omega_{0}}{2}\left(\left|b_{-}\right\rangle\left\langle b_{-}|-| b_{+}\right\rangle\left\langle b_{+}\right|\right)+\dot{\gamma}(t) \sin [\theta(t)]^{2}\left|d_{2}\right\rangle\left\langle d_{2}\right| \\
& +\frac{1}{2} \dot{\gamma}(t) \cos [\theta(t)]^{2}\left(\left|b_{-}\right\rangle\left\langle b_{-}|+| b_{+}\right\rangle\left\langle b_{+}\right|\right)
\end{aligned}
$$

is a diagonal operator which generates the desired adiabatic evolution. In contrast, $\hat{V}_{\mathrm{err}}(t)$ describes nonadiabatic errors in the evolution,

$$
\begin{aligned}
\hat{V}_{\mathrm{err}}(t)= & \frac{\dot{\theta}(t)}{\sqrt{2}}\left(i\left|d_{2}\right\rangle\left\langle b_{-}|-i| d_{2}\right\rangle\left\langle b_{+}\right|+\text {H.c. }\right) \\
& +\frac{\dot{\gamma}(t)}{2}\left[-\cos [\theta(t)]^{2}\left|b_{+}\right\rangle\left\langle b_{-}\left|+\frac{\sin [2 \theta(t)]}{\sqrt{2}}\right| d_{2}\right\rangle\left\langle b_{-}\right|\right. \\
& \left.-\frac{\sin [2 \theta(t)]}{\sqrt{2}}\left|d_{2}\right\rangle\left\langle b_{+}\right|+\text {H.c. }\right] .
\end{aligned}
$$

Equation (13) differs from the nonadiabatic Hamiltonian derived in Ref. [24] because the latter work did not consider STIRAP with time-dependent relative phases.

If one now assumes that we are in the adiabatic limit, i.e., $2 \dot{\theta}(t) / \Omega_{0} \rightarrow 0$ and $2 \dot{\gamma}(t) / \Omega_{0} \rightarrow 0$, then we can ignore $\hat{V}_{\mathrm{err}}(t)$, and the unitary operator describing the evolution is

$$
\begin{aligned}
\hat{U}_{\mathrm{ad}}(t)= & |\tilde{0}\rangle\left\langle\tilde{0}\left|+e^{-i \gamma_{0}}\right| d_{2}\right\rangle\left\langle d_{2}\right| \\
& +e^{i\left(\frac{\Omega_{0}}{2} t-\gamma_{1}\right)}\left|b_{-}\right\rangle\left\langle b_{-}\left|+e^{-i\left(\frac{\Omega_{0}}{2} t+\gamma_{1}\right)}\right| b_{+}\right\rangle\left\langle b_{+}\right| \\
& +\mathcal{O}\left[\frac{\dot{\theta}(t)}{\Omega_{0}}, \frac{\dot{\gamma}(t)}{\Omega_{0}}\right],
\end{aligned}
$$

where

$$
\begin{aligned}
& \gamma_{0}=\int_{0}^{t_{g}} d t_{1} \sin \left[\theta\left(t_{1}\right)\right]^{2} \dot{\gamma}\left(t_{1}\right), \\
& \gamma_{1}=\frac{1}{2} \int_{0}^{t_{g}} d t_{1} \cos \left[\theta\left(t_{1}\right)\right]^{2} \dot{\gamma}\left(t_{1}\right)
\end{aligned}
$$

are the geometric phases accumulated by the dark and bright states, respectively.

Before proceeding, we note that there is an extremely simple choice for the relative control field phase $\gamma(t)$ that, despite first appearances, is compatible with adiabatic evolution. Namely, one can use

$$
\gamma(t)=\gamma_{0} \Theta\left(t-\frac{t_{g}}{2}\right),
$$

where $\Theta(t)$ denotes the Heaviside step function. Despite the discontinuity at $t=t_{g} / 2$, there is no issue with adiabaticity. Note that our chosen pulse shapes [cf. Eq. (2)] satisfy $\theta\left(t_{g} / 2\right)=\pi / 2$, which leads to $\Omega_{a e}\left(t_{g} / 2\right)=0$, implying that the phase $\gamma(t)$ is not well defined at this time; this allows the jump in Eq. (16). Another way to understand that choosing $\gamma(t)$ as given by Eq. (16) does not impact the adiabaticity of the protocol is to realize that the instantaneous eigenenergies of $\hat{H}_{\mathrm{ad}}(t)$ [see Eq. (11)] are not altered by the phase jump. For a more geometric picture, note that this type of control results in a trajectory on the Bloch sphere that resembles a citrus wedge, as depicted in Fig. 1(d). Finally, we emphazise that this choice leads to $\gamma_{1}=0$ [cf. Eq. (15)].

We can express Eq. (14) in the (time-independent) laboratory frame via the transformation $\hat{U}_{\mathrm{ad}}(t)=$ $\hat{S}_{\mathrm{ad}}(t) \hat{U}_{\Lambda, \text { ad }}(t) \hat{S}_{\mathrm{ad}}^{\dagger}(0)$. Using for $\gamma(t)$ Eq. (16), we find at the final time $t=t_{g}$

$$
\hat{U}_{G, \mathrm{ad}}=e^{-i \frac{\gamma_{0}}{2}} e^{-i \frac{\gamma_{0}}{2} \boldsymbol{n} \cdot \hat{\boldsymbol{\sigma}}_{01}} \oplus e^{i \frac{\gamma_{0}}{2}} e^{i \frac{\varphi_{\mathrm{ad}}}{2} \boldsymbol{n}_{\mathrm{ad}} \cdot \hat{\boldsymbol{\sigma}}_{a e}} .
$$

Here, $\quad \hat{\boldsymbol{\sigma}}_{01}=(|0\rangle\langle 1|+$ H.c., $-i|0\rangle\langle 1|+$ H.c., $|0\rangle\langle 0|-| 1\rangle\langle 1|)$ and $\hat{\sigma}_{a e}=(|a\rangle\langle e|+$ H.c., $-i|a\rangle\langle e|+$ H.c., $|a\rangle\langle a|-| e\rangle\langle e|)$ denote a vector of Pauli matrices. We have further defined the unit vectors

$$
\begin{aligned}
\boldsymbol{n} & =[\sin (2 \alpha) \cos (\beta), \sin (2 \alpha) \sin (\beta), \cos (2 \alpha)], \\
\boldsymbol{n}_{\mathrm{ad}} & =\frac{\sin \left(\frac{\Omega_{\mathrm{o}} t_{\mathrm{g}}}{2}\right)}{\sin \left(\frac{\varphi_{\mathrm{ad}}}{2}\right)} \sin \left(\frac{\gamma_{0}}{2}\right)\left[-\cot \left(\frac{\gamma_{0}}{2}\right), 1, \cot \left(\frac{\Omega_{0} t_{g}}{2}\right)\right]
\end{aligned}
$$

and the rotation angle

$$
\varphi_{\mathrm{ad}}=2 \arccos \left[\cos \left(\frac{\gamma_{0}}{2}\right) \cos \left(\frac{\Omega_{0} t_{g}}{2}\right)\right] .
$$

Equation (17) shows clearly that at $t=t_{g}$ the qubit subspace is decoupled from that of the two auxiliary levels. The evolution in the qubit subspace is a simple rotation. The rotation axis $\boldsymbol{n}$ is controlled by the static pulse parameters $\alpha$ and $\beta$, whereas the rotation angle $\gamma_{0}$ is a geometric phase. We thus have a purely geometric arbitrary single-qubit gate. We stress that having a gate that acts independently on the qubit and auxiliary subspaces is crucial: it allows a qubit gate to be performed without first having to prepare the state of the auxiliary levels. 


\section{B. Nonadiabatic errors}

Our goal is to accelerate the adiabatic gate described above. As the first step, we need to understand the effects of nonadiabatic errors that occur when the protocol time is not infinitely slow compared to the inverse instantaneous energy gap $2 / \Omega_{0}$ of $\hat{H}(t)$.

We can calculate nonadiabatic corrections to the evolution perturbatively in $\hat{V}_{\text {err }}(t)$ [cf. Eq. (13)] using a Magnus expansion [35]. Using $\theta(t)$ as defined in Eq. (8) with $P(t)$ given by Eq. (9) and $\gamma(t)$ as defined in Eq. (16), we find to leading order (see Appendix A)

$$
\hat{U}_{G}=\left(e^{-i \frac{\gamma_{0}}{2}} e^{-i \frac{\gamma_{0}}{2} \boldsymbol{n} \cdot \hat{\boldsymbol{\sigma}}} \oplus e^{i \frac{\gamma_{0}}{2}} e^{i \frac{\varphi_{\mathrm{na}}}{2} \boldsymbol{n}_{\mathrm{na}} \cdot \hat{\boldsymbol{\sigma}}_{a e}}\right)+\mathcal{O}\left[\frac{1}{\left(\Omega_{0} t_{g}\right)^{3}}\right],
$$

with

$$
\begin{aligned}
\boldsymbol{n}_{\mathrm{na}} & =\frac{\sin \left[\varphi_{0}\left(t_{g}\right)\right]}{\sin \left(\frac{\varphi_{\mathrm{na}}}{2}\right)} \sin \left(\frac{\gamma_{0}}{2}\right)\left\{-\cot \left(\frac{\gamma_{0}}{2}\right), 1, \cot \left[\varphi_{0}\left(t_{g}\right)\right]\right\} \\
\varphi_{\mathrm{na}} & =2 \arccos \left\{\cos \left(\frac{\gamma_{0}}{2}\right) \cos \left[\varphi_{0}\left(t_{g}\right)\right]\right\} \\
\varphi_{0}\left(t_{g}\right) & =\frac{\Omega_{0} t_{g}}{2}+\frac{10 \pi^{2}}{7 \Omega_{0} t_{g}}
\end{aligned}
$$

Comparing with Eqs. (17) and (20), we see that, to leading order, nonadiabatic errors do not change the nature of the gate: we still have a pure geometric operation on the qubit subspace and the latter is still decoupled from the auxiliary subspace. Nonadiabatic errors only change the rotation performed on the auxiliary levels. The favorable scaling here is a direct consequence of our choice for $\theta(t)$ [cf. Eq. (8)], whose derivatives vanish at the protocol start and end; this corresponds to the "boundary cancellation method" for nonadiabatic error suppression discussed in Refs. [36-38].

A more useful measure for quantifying the impact of nonadiabatic errors is given by the state-averaged fidelity of the gate [39]

$$
\bar{F}=\frac{\operatorname{Tr}\left[\hat{O} \hat{O}^{\dagger}\right]+|\operatorname{Tr}[\hat{O}]|^{2}}{d(d+1)},
$$

where $\operatorname{Tr}[\cdot]$ denotes the trace operation, $d=4$ is the dimension of the Hilbert space, and $\hat{O}=\hat{U}_{G, \text { ad }}^{\dagger} \hat{U}_{G}$, where $\hat{U}_{G \text {, ad }}$ is given in Eq. (17) and $\hat{U}_{G}$ is the unitary operator generated by Eq. (1) evaluated at $t=t_{g}$. Using the approximate $\hat{U}_{G}$ given in Eq. (20), we find

$$
\bar{F}=1-\frac{40 \pi^{4}}{49\left(\Omega_{0} t_{g}\right)^{2}}+\mathcal{O}\left[\frac{1}{\left(\Omega_{0} t_{g}\right)^{4}}\right] .
$$

However, it is misleading to compare $\hat{U}_{G \text {, ad }}$ to $\hat{U}_{G}$ to determine the gating time for which the error $\varepsilon=1-\bar{F}$ goes above a critical threshold, e.g., $\varepsilon=10^{-3}$ for quantum error correction. In Eq. (23) the deviations from unity are only due to imperfect dynamics in the auxiliary subspace as we identified earlier [see Eqs. (17) and (20)]. Since Eq. (22) holds for any linear operator $\hat{O}$ in a $d$-dimensional Hilbert space, we consider instead $\hat{O}_{q}=\hat{P}_{q} \hat{U}_{G \text {,ad }}^{\dagger} \hat{P}_{q} \hat{U}_{G} \hat{P}_{q}$, which allows us to quantify errors that only affect the dynamics in the qubit subspace. Here, $\hat{P}_{q}$ is the projector onto the qubit subspace. $\hat{O}_{q}$ corresponds to measuring the overlap between the unitary

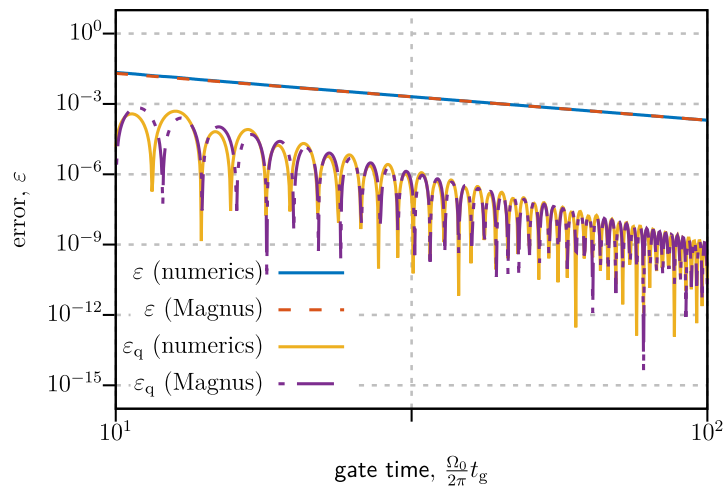

FIG. 2. Error $\varepsilon=1-\bar{F}$ [see Eq. (22)] as a function of the gate time for the unitary operator $\hat{U}_{G}$ acting on both the qubit and auxiliary subspace and its projection $\hat{U}_{q}$ on the qubit subspace $\left(\varepsilon_{q}\right)$. Corrections to the ideal gate due to nonadiabatic transitions only affect the auxiliary subspace at leading order in $\hat{V}_{\text {err }}$, while the dynamics in the qubit subspace is only affected at third order. This is a consequence of our choice of $\theta(t)$, whose first and second derivatives vanish at $t=0, t_{g} / 2$, and $t_{g}$.

operator $\hat{U}_{q, \text { ad }}=\hat{P}_{q} \hat{U}_{G, \text { ad }} \hat{P}_{q}$ and the operator $\hat{U}_{q}=\hat{P}_{q} \hat{U}_{G} \hat{P}_{q}$; the latter is not necessarily unitary. Due to the direct sum structure of $\hat{U}_{G \text {,ad }}$ [cf. (17)], the projection operation yields the ideal gate acting on the qubit subspace only.

Within this framework, and performing a fourth-order Magnus expansion (see Appendix A), we find

$\bar{F}_{q}=1+\frac{a \pi^{2}}{\left(\Omega_{0} t_{g}\right)^{6}}\left[-1+\cos \left(\frac{\Omega_{0} t_{g}}{4}\right) \cos \left(\gamma_{0}\right)\right] \sin ^{2}\left(\frac{\Omega_{0} t_{g}}{8}\right)$,

with $a=14745600$. We stress that there exist special gate times for which the fidelity is equal to unity. Using Eq. (24), we find those times to be

$$
t_{g}= \begin{cases}\frac{8 \pi}{\Omega_{0}} k_{1}, & k_{1} \in \mathbb{N}^{*}, \\ \frac{4\left(\pi+2 \pi k_{2}\right)}{\Omega_{0}}, & k_{2} \in \mathbb{N} .\end{cases}
$$

This phenomenon (a coherent cancellation of nonadiabatic effects) has also been discussed in Ref. [38].

In Fig. 2, we plot the error $\varepsilon=1-\bar{F}$ [see Eq. (22)] as a function of the gate time for $\hat{O}$ and $\hat{O}_{q}$ obtained both numerically by integrating the Schrödinger equation and perturbatively via the Magnus expansion. In the adiabatic regime, i.e., $\Omega_{0} t_{g} / 2 \rightarrow \infty$, the Magnus expansion fully captures deviations from the ideal adiabatic evolution, which confirms our error scaling analysis. However, even with the improved error scaling in the qubit subspace, the achievable gate times in a realistic setup remain longer than the typical decoherence rates.

\section{ACCELERATED GATE}

We now turn to the main goal of this paper: How can we accelerate the geometric qubit gate presented in Sec. II A using the general philosophy of "shortcuts to adiabaticity" [10-12,24,40]? At first glance, this is a nontrivial problem. The original purpose of STAs is to accelerate the evolution of a specific initial state, and usually, one does not care about 
the final, overall phase of the state. In contrast, we want to accelerate the evolution of an arbitrary initial qubit state, and the phases acquired by the adiabatic states are of crucial importance. Despite these difficulties, we show that our desired goal can indeed be accomplished. We use our recently proposed dressed-state approach, which allows acceleration of STIRAP-type processes by simply modifying the form of the original control pulses [19,24]. Note that Ref. [30] did not consider these difficulties; as we show below, it is not a trivial task to find a suitable STA that preserves the phases acquired by the adiabatic states.

Our goal will be to modify the three control fields $\Omega_{i e}(t)$ $(i=0,1, a)$ from the values given in Eqs. (2) and (8), so that the desired gate operation is accomplished even though the total protocol time $t_{g}$ is not long compared to $2 / \Omega_{0}$. This modification can be described by adding a term to the Hamiltonian, i.e., $\hat{H}(t) \rightarrow \hat{H}(t)+\hat{W}(t) \equiv \hat{H}_{\text {mod }}(t)$. In the adiabatic frame, this modification can be written as

$$
\begin{aligned}
\hat{W}_{\mathrm{ad}}(t)= & \frac{1}{2}\left[-W_{z}(t)\left(\left|b_{-}\right\rangle\left\langle b_{-}|-| b_{+}\right\rangle\left\langle b_{+}\right|\right)\right. \\
& +\frac{W_{x}(t)}{\sqrt{2}}\left(\left|d_{2}\right\rangle\left\langle b_{-}|+| d_{2}\right\rangle\left\langle b_{+}\right|+\text {H.c. }\right) \\
& \left.+\frac{W_{y}(t)}{\sqrt{2}}\left(-i\left|b_{-}\right\rangle\left\langle d_{2}|-i| b_{+}\right\rangle\left\langle d_{2}\right|+\text { H.c. }\right)\right] .
\end{aligned}
$$

One can readily verify that transforming Eq. (26) to the original frame, $\hat{W}(t)=\hat{S}_{\text {ad }}(t) \hat{W}_{\text {ad }}(t) \hat{S}_{\text {ad }}^{\dagger}(t)$, results in a control Hamiltonian having the same form as Eq. (1); no additional control fields are required. Note that the qubit-only dark state $|\tilde{0}\rangle$ remains decoupled for any choice of the $W_{j}(t), j \in$ $\{x, y, z\}$.

Conventional STAs attempt to modify $\hat{H}(t)$ so that the evolution follows the (original) adiabatic trajectory at all times. In contrast, the dressed-state approach of Ref. [24] aims for something less extreme. We let the system deviate from the adiabatic trajectory at intermediate times. This can be framed as a time-dependent dressing of the original adiabatic eigenstate, with the dressing vanishing at $t=0$ and $t=t_{g}$. For our problem, we need to add an additional constraint. We must find a dressed version of the dark state $\left|d_{2}\right\rangle$,

$$
\left|\tilde{d}_{2}(t)\right\rangle \equiv \hat{S}_{\mu}^{\dagger}(t)\left|d_{2}\right\rangle,
$$

which retains the geometric nature of the evolution. In other words, we require that the new dressed dark state does not acquire any dynamical phases.

\section{A. Generic dressing}

Following Ref. [24] we try a simple dressing transformation,

$$
\hat{S}_{\mu}(t)=\exp \left[-i \mu(t)\left(\left|b_{-}\right\rangle\left\langle d_{2}\right|+\text { H.c. }\right)\right],
$$

where the dressing angle $\mu(t)$ remains to be determined. It must satisfy $\mu(0)=\mu\left(t_{g}\right)=0$, to ensure that the dressing vanishes at the start and end of the protocol (which then guarantees $\left|\tilde{d}_{2}(t)\right\rangle=|\tilde{1}\rangle$ at $t=0$ and $t=t_{g}$ ). Note that at this stage we only assume $\theta(t)$ to be of the form given in Eq. (8), while no particular form is assumed for $\gamma(t)$.
The goal is now to pick the dressing parameter $\mu(t)$ and control modifications $\hat{W}(t)$ such that the resulting dynamics does not cause transitions out of the dressed dark state. By considering the dynamics in the time-dependent frame defined by Eq. (28) (dressing frame), we find that this can be accomplished by choosing the dressing angle to fulfill the differential equation

$$
\dot{\mu}(t)=\frac{\sin [2 \theta(t)] \dot{\gamma}(t)}{\sqrt{2}}
$$

and the control fields to be

$$
\begin{aligned}
W_{x}(t)= & \sin [2 \theta(t)] \dot{\gamma}(t), \\
W_{y}(t)= & \sqrt{2}\left[\cos [\theta(t)]^{2} \tan [\mu(t)] \dot{\gamma}(t)+\sqrt{2} \dot{\theta}(t)\right], \\
W_{z}(t)= & -\Omega_{0}+4 \sqrt{2} \cot [2 \mu(t)] \dot{\theta}(t) \\
& +\frac{\dot{\gamma}(t)}{2}\left[1+5 \cos [2 \theta(t)]-2 \cos [\theta(t)]^{2} \sec [\mu(t)]^{2}\right] .
\end{aligned}
$$

Unfortunately, suppressing unwanted transitions is not enough to achieve our gate. We also need control over the phase acquired by the dressed dark state. In particular, it should not acquire a dynamical phase which depends explicitly on $t_{g}$ while still accumulating a geometrical phase. We stress that the control fields in Eq. (30) also cancel the purely dynamical phase originating from the dressing by ensuring that the energy of the dressed dark state is 0 . Within this framework the phase accumulated by the dressed dark state is given by

$$
\begin{aligned}
\varphi_{\mathrm{dds}}= & \int_{0}^{t_{g}} d t \frac{\sec [\mu(t)]^{2}}{8}\{\dot{\gamma}(t)[3+\cos [2 \mu(t)] \\
& -\cos [2 \theta(t)](1+3 \cos [2 \mu(t)])] \\
& +4 \sqrt{2} \sin [2 \mu(t)] \dot{\theta}(t)\} .
\end{aligned}
$$

In spite of the similarities to Eq. (15), there is no guarantee that this phase is purely geometric since one might not be able to express $\mu$ as a function of $\gamma$ and $\theta$ only. In our example, however, the situation is far worse. A solution of Eq. (29) that fulfills the requirement that the dressing must vanish at the boundaries, $\left[\mu(0)=\mu\left(t_{g}\right)=0\right]$, leads to $\dot{\gamma}(t)$ being an antisymmetric function in the interval $\left[0, t_{g}\right] \operatorname{since} \sin [2 \theta(t)]$ is symmetric in said interval [see Eq. (8)]. Using the symmetry of the functions involved in Eq. (31), one finds $\varphi_{\mathrm{dds}}=0$.

\section{B. Spin-based dressing}

However, for $\gamma(t)$ given by Eq. (16) and arbitrary $\theta(t)$ of the form in Eq. (8), one can find a large class of STAs for which there is an accumulated phase whose nature is geometric. To proceed, we first define effective spin- 1 operators to describe the dressed frame states: $\hat{J}_{x}=\left(\left|\tilde{d}_{2}\right\rangle\left\langle\tilde{b}_{+}|+| \tilde{d}_{2}\right\rangle\left\langle\tilde{b}_{-}\right|+\right.$ H.c. $) / \sqrt{2}, \hat{J}_{y}=\left(i\left|\tilde{d}_{2}\right\rangle\left\langle\tilde{b}_{-}|-i| \tilde{d}_{2}\right\rangle\left\langle\tilde{b}_{+}\right|+\right.$H.c. $) / \sqrt{2}$, and $\hat{J}_{z}=$ $\left(\left|\tilde{b}_{-}\right\rangle\left\langle\tilde{b}_{-}|-| \tilde{b}_{+}\right\rangle\left\langle\tilde{b}_{+}\right|\right)$. The dressing transformation of interest is then

$$
\hat{S}_{v}(t)=\exp \left[-i v(t) \hat{J}_{x, \text { ad }}\right]
$$


In the dressed frame, the Hamiltonian is given by

$$
\begin{aligned}
\hat{H}_{\text {dressed }} & =\hat{S}_{v}^{\dagger}(t) \hat{H}_{\mathrm{ad}}(t) \hat{S}_{v}(t)-i \hat{S}_{v}^{\dagger}(t) \partial_{t} \hat{S}_{v}^{\dagger}(t) \\
& =\hat{H}_{\mathrm{spin}}(t)+\hat{H}_{\mathrm{ns}}(t)+\hat{H}_{\mathrm{geom}}(t),
\end{aligned}
$$

with

$$
\begin{aligned}
& \hat{H}_{\text {spin }}(t)=B_{z}(t) \hat{J}_{z}+B_{x}(t) \hat{J}_{x}+B_{y}(t) \hat{J}_{y} \\
\hat{H}_{\mathrm{ns}}(t)= & \frac{\dot{\gamma}(t)}{2}\left[\Xi_{1}(t)\left(\left|\tilde{b}_{-}\right\rangle\left\langle\tilde{b}_{-}|+| \tilde{b}_{+}\right\rangle\left\langle\tilde{b}_{+}\right|\right)\right. \\
& +\Xi_{2}(t)\left(\left|\tilde{b}_{+}\right\rangle\left\langle\tilde{b}_{-}\right|+\text {H.c. }\right)+\Xi_{3}(t)\left(i\left|\tilde{b}_{+}\right\rangle\left\langle\tilde{b}_{-}\right|+\text {H.c. }\right) \\
& \left.+\Xi_{4}(t)\left(\left|\tilde{d}_{2}\right\rangle \tilde{b}_{-}|-| \tilde{d}_{2}\right\rangle\left\langle\tilde{b}_{+}\right|+\text {H.c. }\right) \\
& \left.+\Xi_{5}(t)\left(i\left|\tilde{d}_{2}\right\rangle\left\langle\tilde{b}_{-}|+i| \tilde{d}_{2}\right\rangle\left\langle\tilde{b}_{+}\right|+\text {H.c. }\right)\right],
\end{aligned}
$$

and

$$
\hat{H}_{\text {geom }}(t)=\dot{\gamma}(t) \sin ^{2}[\theta(t)] \cos ^{2}[v(t)]\left|\tilde{d}_{2}\right\rangle\left\langle\tilde{d}_{2}\right| .
$$

We have written Eq. (33) as the sum of a spin Hamiltonian [see Eq. (34)], a nonspin Hamiltonian [see Eq. (35)], and a Hamiltonian that generates the geometric phase [see Eq. (36)]. We also have defined the effective magnetic field components

$$
\begin{aligned}
& B_{x}(t)=-\dot{v}(t), \\
& B_{y}(t)=-\frac{\Omega_{0}}{2} \sin [v(t)]+\dot{\theta}(t) \cos [v(t)], \\
& B_{z}(t)=-\frac{\Omega_{0}}{2} \cos [v(t)]-\dot{\theta}(t) \sin [v(t)],
\end{aligned}
$$

as well as the parameters of the non-spin Hamiltonian,

$$
\begin{aligned}
& \Xi_{1}(t)=\cos ^{2}[\theta(t)]+\sin ^{2}[\theta(t)] \sin ^{2}[v(t)], \\
& \Xi_{2}(t)=-\cos ^{2}[\theta(t)]+\sin ^{2}[\theta(t)] \sin ^{2}[v(t)], \\
& \Xi_{3}(t)=\sin [2 \theta(t)] \sin [v(t)], \\
& \Xi_{4}(t)=\frac{\sin [2 \theta(t)] \cos [v(t)]}{\sqrt{2}}, \\
& \Xi_{5}(t)=-\frac{\sin ^{2}[\theta(t)] \sin [2 v(t)]}{\sqrt{2}} .
\end{aligned}
$$

The choice of dressing in Eq. (32) was made to ensure that $\left\langle\tilde{d}_{2}\left|\hat{H}_{\text {dressed }}(t)-\hat{H}_{\text {geom }}(t)\right| \tilde{d}_{2}\right\rangle=0$; this partly solves the problem of the STA giving rise to unwanted dynamical phases in the evolution of the dressed dark state $\left|\tilde{d}_{2}\right\rangle$. In contrast to the STA approach (see Sec. III A), we do not start by looking for a dressing angle $v(t)$ that generates a dressing transformation that cancels unwanted transitions (between dressed dark state and dressed bright states). Instead, we start by looking for a $v(t)$ that gives a specific value for the phase accumulated by the dressed dark state $\left|\tilde{d}_{2}\right\rangle$. Neglecting for a moment transitions involving the dressed dark state, we have that the phase accumulated by $\left|\tilde{d}_{2}\right\rangle$ is given by

$$
\varphi_{\mathrm{dds}}=\int_{0}^{t_{g}} d t \sin ^{2}[\theta(t)] \cos ^{2}[v(t)] \dot{\gamma}(t) .
$$

Taking into account that we have chosen a particular form for $\gamma(t)$ [see Eq. (16)] and comparing Eq. (39) to Eq. (15), we see that the phase accumulated by the dressed dark state is equal to the adiabatic-limit dark-state geometric phase $\gamma_{0}$ if

$$
v\left(t_{g} / 2\right)=0,
$$

i.e., we must restrict ourselves to a class of dressing transformations that exactly vanish halfway through the protocol.

There is a second consequence of having to work with dressing transformations that fulfill Eq. (40): one can easily verify that $\hat{H}_{\mathrm{ns}}(t)$ does not generate any dynamics. Since $\dot{\gamma}(t)=\gamma_{0} \delta\left(t-t_{g} / 2\right)$, integrating $\hat{H}_{\mathrm{ns}}(t)$ between $t=0$ and $t=t_{g}$ yields 0 because we evaluate $\hat{H}_{\mathrm{ns}}(t)$ at $t=t_{g} / 2$, where $\theta\left(t_{g} / 2\right)=\pi / 2$ and $v\left(t_{g} / 2\right)=0$, such that all parameters defined in Eq. (38) evaluate to 0 .

Within this framework all that remains is to find a specific dressing function $v(t)$ satisfying Eq. (40) and a corresponding control Hamiltonian $\hat{W}(t)$ that cancels unwanted transitions generated by $\hat{H}_{\text {spin }}(t)$. This is essentially equivalent to the general problem treated in Ref. [24]. While many choices are possible, a particular simple approach is the so-called superadiabatic transitionless driving dressing introduced in Ref. [24]. This is defined by the specific dressing angle

$$
v(t)=v_{\mathrm{SATD}}(t)=\arctan \left[\frac{2 \dot{\theta}(t)}{\Omega_{0}}\right] .
$$

This satisfies our constraint, Eq. (40), as long as the initial pulse sequence satisfies $\dot{\theta}\left(t_{g} / 2\right)=0$. For example, the pulse shape in Eq. (9) satisfies this property.

Before proceeding we note that the phase accumulated by the dressed dark state can still be viewed as a geometric phase. As long as Eq. (40) is fulfilled the accumulated phase is independent of the protocol time $t_{g}$ and does not depend on the details of the pulse. We stress, however, one more time that the dressing transformation allowing one to get an STA that preserves the geometric nature of the phase accumulated by the dressed dark state $\left|\tilde{d}_{2}(t)\right\rangle$ explicitly depends on our choice of $\gamma(t)$ and that our specific choice of dressing [see Eq. (41)] further requires the adiabatic protocol to obey $\dot{\theta}\left(t_{g} / 2\right)=0$.

For this choice of dressing, the required modified control fields (which cancel transitions out of the dressed dark state) are

$$
\begin{aligned}
& \Omega_{0 e}(t) \rightarrow \frac{\Omega_{0}}{2} \cos (\alpha)\left[\sin [\theta(t)]+4 \frac{\cos [\theta(t)] \ddot{\theta}(t)}{\Omega_{0}^{2}+4 \dot{\theta}^{2}(t)}\right] \\
& \Omega_{1 e}(t) \rightarrow \frac{\Omega_{0}}{2} \sin (\alpha) e^{i \beta}\left[\sin [\theta(t)]+4 \frac{\cos [\theta(t)] \ddot{\theta}(t)}{\Omega_{0}^{2}+4 \dot{\theta}^{2}(t)}\right] \\
& \Omega_{a e}(t) \rightarrow \frac{\Omega_{0}}{2} e^{i \gamma(t)}\left[\cos [\theta(t)]-4 \frac{\sin [\theta(t)] \ddot{\theta}(t)}{\Omega_{0}^{2}+4 \dot{\theta}^{2}(t)}\right]
\end{aligned}
$$

Combining these results, we find that the accelerated dynamics results in an evolution that at $t=t_{g}$ yields the gate

$$
\hat{U}_{G, \mathrm{SATD}}=e^{-i \frac{\gamma_{0}}{2}} e^{-i \frac{\gamma_{0}}{2} \boldsymbol{n} \cdot \hat{\boldsymbol{\sigma}}} \oplus e^{i \frac{\gamma_{0}}{2}} e^{i \frac{\varphi_{\mathrm{SATD}}}{2} \boldsymbol{n}_{\mathrm{SATD}} \cdot \hat{\boldsymbol{\sigma}}_{a e}},
$$

whose action on the qubit subspace is the same as $\hat{U}_{G \text {, ad }}$ [see Eq. (17)] but acts differently on the auxiliary subspace. The latter undergoes a rotation of angle $\varphi_{\text {SATD }}=$ $2 \arccos \left[\cos \left(\gamma_{0} / 2\right) \cos (\Phi)\right]$ around the axis

$$
\boldsymbol{n}_{\mathrm{SATD}}=\frac{\sin (\Phi) \sin \left(\frac{\gamma_{0}}{2}\right)}{\sin \left(\frac{\varphi_{\mathrm{SATD}}}{2}\right)}\left[-\cot \left(\frac{\gamma_{0}}{2}\right), 1, \cot (\Phi)\right],
$$

with $\Phi=\int_{0}^{t_{g} / 2} d t \sqrt{\Omega_{0}^{2}+4 \dot{\theta}^{2}(t)}$. 
We note that Eq. (43) always leads to a perfect qubitsubspace fidelity $\bar{F}_{q}=1$ independent of the speed of the protocol.

\section{DISSIPATIVE DYNAMICS}

In the following section, we characterize the performance of both the adiabatic and the STA gates in the presence of imperfections. We consider two types of imperfections: dissipation and uncertainties in the parameters of the Hamiltonian.

To model the loss we consider a Lindblad master equation that describes pure dephasing of the ground and excited states

$$
\begin{aligned}
\partial_{t} \hat{\rho}(t)= & -i[\hat{H}(t), \hat{\rho}(t)]+\sum_{j=0,1, a, e} \Gamma_{\varphi, j} \\
& \times\left[|j\rangle\langle j|\hat{\rho}(t)| j\rangle\langle j|-\frac{1}{2}\{|j\rangle\langle j|, \hat{\rho}(t)\}_{+}\right],
\end{aligned}
$$

where $\hat{H}(t)$ is the Hamiltonian, $\hat{\rho}(t)$ is the density operator of the system, $\Gamma_{\varphi, j}(j \in\{0,1, a, e\})$ is the dephasing rate of state $|j\rangle$, and we have defined the anticommutator $\left\{\hat{O}_{1}, \hat{O}_{2}\right\}_{+}=$ $\hat{O}_{1} \hat{O}_{2}+\hat{O}_{2} \hat{O}_{1}$. We stress that in the adiabatic frame the dephasing processes we are considering lead to transitions between instantaneous eigenstates. For this reason we do not explicitly consider relaxation processes.

To quantify how decoherence affects the performance of the gate, we use the result of Bowdrey et al. for the average fidelity of single-qubit maps [41],

$$
\bar{F}_{\text {map }}=\frac{1}{6} \sum_{j= \pm x, \pm y, \pm z} \operatorname{Tr}\left[\hat{U}_{q} \hat{\rho}_{j} \hat{U}_{q}^{\dagger} \hat{\rho}_{j}(t)\right]
$$

where $\hat{\rho}_{j}$ with $j \in\{ \pm x, \pm y, \pm z\}$ is an axial pure state on the Bloch sphere of the qubit, e.g., $\hat{\rho}_{x}=1 / 2(|0\rangle+|1\rangle)(\langle 0|+$ $\langle 1|), \hat{U}_{q}$ is defined earlier in the text, and $\hat{\rho}_{j}(t)$ is a solution of Eq. (45) for the initial state $\hat{\rho}_{j}$.

In addition to errors due to noise, we also consider errors arising from uncertainties in the system Hamiltonian. Here, we assume that the amplitude parameter $\Omega_{0}$ [cf. Eqs. (1) and Eq. (2)] is only known with finite precision, as described by the probability distribution $p\left(\Omega_{0}\right)$. In the following we assume $p\left(\Omega_{0}\right)$ to be uniform in the interval $\left[\Omega_{0}(1-k), \Omega_{0}(1+k)\right]$ with $k \in(0,1)$. The performance of the gate is then quantified via the averaged average fidelity,

$$
\left\langle\bar{F}_{\text {map }}\right\rangle=\int_{\Omega_{0}(1-k)}^{\Omega_{0}(1+k)} d \Omega_{0} p\left(\Omega_{0}\right) \bar{F}_{\text {map }}\left(\Omega_{0}\right) .
$$

For the results that follow, accelerated gates are implemented using a starting pulse shape given by Eq. (9) and the SATD STA defined by Eqs. (41) and (42).

\section{A. Excited-state dephasing}

In Fig. 3, we plot the error $\varepsilon_{\text {map }}=1-\bar{F}_{\text {map }}$ as a function of the gate time for $\alpha=\pi / 4, \beta=0, \gamma_{0}=\pi, \Gamma_{\varphi, e}=\Omega_{0} /(2 \pi \times$ $100)$, and $\Gamma_{\varphi, j}=0$ for $j \in\{0,1, a\}$ for both the adiabatic (blue traces) and the accelerated (orange traces) protocols, either without uncertainty on $\Omega_{0}$ (dashed traces) or with an uncertainty of $40 \%(k=0.2)$ on $\Omega_{0}$ (solid traces). We have indicated by the green arrow the shortest gate time for which the maximal amplitude of the modified controls is still

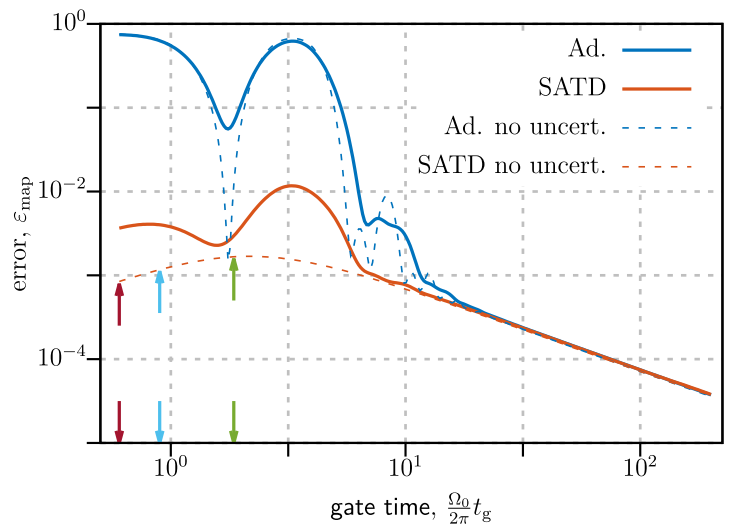

FIG. 3. Comparison of the average error $\varepsilon_{\text {map }}=1-\bar{F}_{\text {map }}$ between adiabatic (upper blue traces) and accelerated (lower orange traces) gates either without uncertainty on $\Omega_{0}$ (dashed traces) or with $40 \%(k=0.2)$ uncertainty on $\Omega_{0}$ (solid traces) as a function of the gate time for $\alpha=\pi / 4, \beta=0, \gamma_{0}=\pi, \Gamma_{\varphi, e}=10^{-2}\left(\Omega_{0} / 2 \pi\right)$, and $\Gamma_{\varphi, j}=0$ for $j \in\{0,1, a\}$. Gate times shorter than the one indicated by the green arrow (first arrow from the right) result in a modified control sequence whose maximal amplitude is higher than $\Omega_{0}$. The blue (second from the right) and red (third from the right) arrows indicate a modified control sequence that requires twice and three times the energy cost [see Eq. (48)] of the adiabatic sequence, respectively.

$\Omega_{0}$ and by the blue (red) arrow the gate time for which the energy cost to generate the STA is twice (three times) as high as the energy cost to generate the adiabatic control sequence. We define the energy cost of a control sequence as

$$
C=\frac{1}{t_{g}} \int_{0}^{t_{g}} d t\|\hat{H}(t)\|_{2},
$$

where $\|\hat{H}(t)\|_{2}=\sigma_{\max }[\hat{H}(t)]$ is the $p=2$ operator norm equal to the largest singular value of the Hamiltonian operator $\hat{H}(t)$ denoted by $\sigma_{\max }[\hat{H}(t)]$.

We start by observing that going to the adiabatic regime, $t_{g} \rightarrow \infty$, results in both gates becoming insensitive to noise and uncertainty. This is expected because the evolution of the system can be reduced to the evolution of the dark state $\left|d_{2}(t)\right\rangle$ [see Eq. (6)] which contains no excited-state amplitude. We note that for the accelerated gate, going to the adiabatic regime results in a vanishing dressing such that the dressed dark state is effectively $\left|d_{2}\right\rangle$. Moreover, a small uncertainty in the instantaneous gap is irrelevant as long as $t_{g} \gg 2 / \Omega_{0}$. Only outside of the adiabatic regime are both gates affected by a lossy excited state since during the evolution the excited state will be occupied. One clearly sees that the accelerated version of the gate outperforms its adiabatic counterpart; this reflects the fact that the mechanism leading to excited-state occupancy is different in each case.

For the adiabatic version of the gate, nonadiabatic processes are responsible for the transitions between the dark state $\left|d_{2}\right\rangle$ and the bright states $\left|b_{ \pm}\right\rangle$, which contain a finite excited-state amplitude [see Eq. (7)]. Since nonadiabatic transitions first need to happen for noise in the excited state to disrupt the dynamics, the gate error is mainly dominated by nonadiabatic errors. However, signatures of the dissipative dynamics can be observed for the special times [see Eq. (25)] 
for which the coherent evolution brings the system back to the dark state. These special times no longer lead to a perfect gate because the coherent mechanism that brings the system back to the dark state is disrupted by excited-state dephasing. This coherent mechanism is further hindered by the uncertainty in $\Omega_{0}$.

The accelerated gate is constructed such that whatever amplitude leaves the dark state it has to return to the dark state by the end of the protocol; this is equivalent to having the system remain in the dressed dark state $\left|\tilde{d}_{2}(t)\right\rangle$ for the whole evolution. However, leaving the dark state is not harmless in the presence of a noisy excited state: it disrupts the STA dynamics in two ways. First, there is no guarantee that the amplitude leaving the dark state returns by the end of the evolution. Second, even if the amplitude that left the dark state returns, it could come back with a phase error. These two mechanisms can be identified as the leading-order processes leading to deviations of the average fidelity [Eq. (46)] from unity. Using a Magnus expansion we can find approximate solutions of Eq. (45) which we use to evaluate $\bar{F}_{\text {map }}$ [Eq. (46)] (see Appendix B). We find

$$
\begin{aligned}
\bar{F}_{\text {map }}= & 1-\frac{4}{3} \Gamma_{\varphi, e} \int_{0}^{t_{g} / 2} d t \frac{\dot{\theta}^{2}(t)}{\Omega_{0}^{2}+4 \dot{\theta}^{2}(t)}-\frac{8}{3} \Gamma_{\varphi, e} \Omega_{0}^{2} \\
& \int_{0}^{t_{g} / 2} d t \frac{\dot{\theta}^{2}(t)}{\left[\Omega_{0}^{2}+4 \dot{\theta}^{2}(t)\right]^{2}}+\mathcal{O}\left[\left(\frac{\Gamma_{\varphi, e}}{\Omega_{0}^{2} t_{g}}\right)^{2}\right],
\end{aligned}
$$

which is in good agreement with numerical simulations (see Appendix B). In particular, Eq. (49) captures the nonmonotonic behavior of the gate error as a function of the gate time. Sufficiently faster gates become insensitive to a lossy excited state. We note, however, that reaching such a regime experimentally is difficult.

In the presence of uncertainty in $\Omega_{0}$, it is impossible to realize the exact STA that would cancel out nonadiabatic transitions. As a result, a low nonadiabatic transition probability from the dressed dark state to the bright states remains. This becomes apparent for faster gate times where the fidelity of the accelerated gates oscillates in sync with the fidelity of the adiabatic gate.

Finally, we note that in this scenario if being fast is not essential, then there is no benefit in using an accelerated gate. Both gates perform equally well in the adiabatic regime. However, if the gate time has to be below a threshold outside of the adiabatic regime, then the accelerated gate will outperform the adiabatic one.

\section{B. Ground- and excited-state dephasing}

In Fig. 4 , we plot the error, $\varepsilon_{\text {map }}=1-\bar{F}_{\text {map }}$, as a function of the gate time for $\alpha=\pi / 4, \beta=0, \gamma_{0}=\pi, \Gamma_{\varphi, e}=$ $\Omega_{0} /(2 \pi \times 100)$, and $\Gamma_{\varphi, 0}=\Gamma_{\varphi, 1}=\Gamma_{\varphi, a}=\Omega_{0} /(2 \pi \times 100)$ for both the adiabatic (blue traces) and the accelerated (orange traces) protocols, either without uncertainty in $\Omega_{0}$ (dashed traces) or with an uncertainty of $40 \%(k=0.2)$ in $\Omega_{0}$ (solid traces). Similarly to Fig. 3, we have indicated by the green arrow the shortest gate time for which the maximal amplitude of the modified controls is still $\Omega_{0}$ and by the blue (red) arrow the gate time for which the energy cost [see Eq. (48)] to

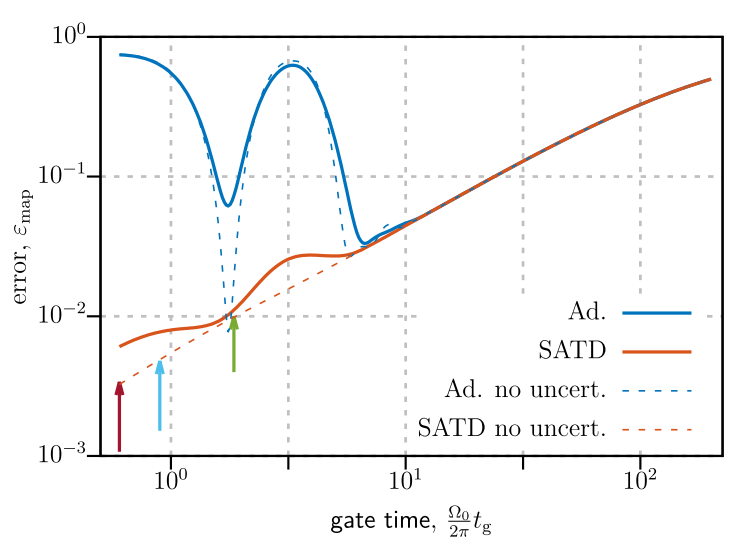

FIG. 4. Comparison of the average error $\varepsilon_{\text {map }}=1-\bar{F}_{\text {map }}$ between adiabatic (upper blue traces) and accelerated (lower orange traces) gates either without uncertainty on $\Omega_{0}$ (dashed traces) or with $40 \%(k=0.2)$ uncertainty on $\Omega_{0}$ (solid traces) as a function of the gate time for $\alpha=\pi / 4, \beta=0, \gamma_{0}=\pi, \Gamma_{\varphi, e}=10^{-2}\left(\Omega_{0} / 2 \pi\right)$, and $\Gamma_{\varphi, j}=10^{-2}\left(\Omega_{0} / 2 \pi\right)$ for $j \in\{0,1, a\}$. Gate times shorter than the one indicated by the green arrow (first from the right) result in a modified control sequence whose maximal amplitude is higher than $\Omega_{0}$. The blue (second from the right) and red (third from the right) arrows indicate modified control sequences that require twice and three times the energy cost [see Eq. (48)] of the adiabatic sequence, respectively.

generate the STA is twice (three times) as high as the energy cost to generate the adiabatic control sequence.

In contrast to the case where only the excited state is lossy, operating in the adiabatic regime does not lead to a perfect gate. The dephasing on the ground-state manifold sets a threshold for the slowest "allowed" gate time. As a consequence, the adiabatic version of the gate becomes an unviable option. Trying to perform the gate more rapidly to avoid ground-state dephasing unavoidably leads to a regime where the dominating source of errors are nonadiabatic transitions. On the other hand, the accelerated gate is less susceptible to nonadiabatic errors. As a result decreasing the gate time allows one to escape the interval for which the error is mainly dominated by ground-state dephasing, $t_{g} \ll 1 /\left(\Gamma_{\varphi, 0}+\right.$ $\left.\Gamma_{\varphi, 1}+\Gamma_{\varphi, a}\right)$, to operate in a regime where only excited-state dephasing contributes to the gate error.

In this scenario, being fast becomes essential and corresponds to a situation where the accelerated gate provides a real benefit over its adiabatic counterpart.

\section{Extended robustness comparison}

To identify the regimes where the accelerated gate provides a clear benefit over the adiabatic gate, we look for the gate time that yields the smallest error for fixed dephasing rates. We have constrained the minimal gate time by imposing that the maximal amplitude of the modified pulse sequence cannot be higher than $\Omega_{0}$. For simplicity we consider the case where the ground-state dephasing rates are equal, i.e., $\Gamma_{\varphi, 0}=$ $\Gamma_{\varphi, 1}=\Gamma_{\varphi, a}=\Gamma_{\varphi, \mathrm{GS}}$. We also assume that the uncertainty on $\Omega_{0}$ is $10 \%(k=0.05)$. In Fig. 5, we plot contour lines for different error thresholds as a function of $\Gamma_{\varphi, \mathrm{GS}}$ and $\Gamma_{\varphi, e}$ for the adiabatic gate (dashed lines) and accelerated gate (solid 


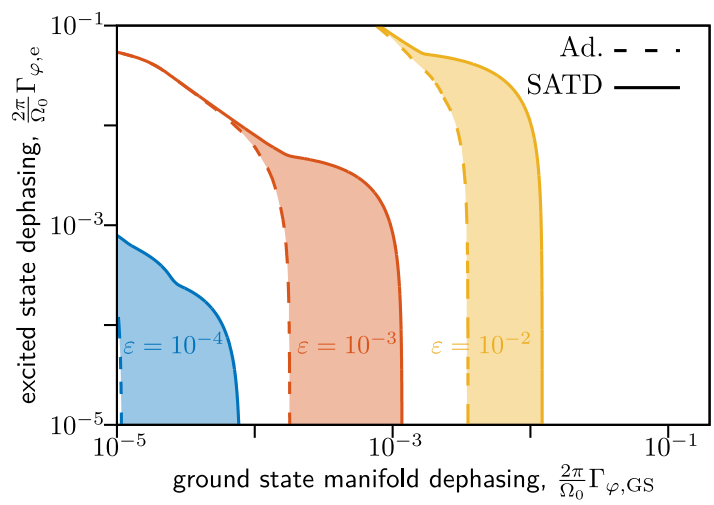

FIG. 5. Comparison of the smallest gate error between adiabatic and accelerated gates. For fixed values of the dephasing rates we look for the gate time that yields the smallest error. We consider an uncertainty on $\Omega_{0}$ of $10 \%(k=0.05)$ and $\Gamma_{\varphi, 0}=\Gamma_{\varphi, 1}=\Gamma_{\varphi, a}=$ $\Gamma_{\varphi, \text { GS }}$. The accelerated gate reaches the same error as the adiabatic gate for dephasing rates that are roughly one order of magnitude larger.

lines). For the displayed contours, we see that the accelerated gate reaches the same error level as the adiabatic gate for rates that can be roughly up to one order of magnitude higher.

\section{CONCLUSION}

We have shown how to use the framework of shortcuts to adiabaticity to accelerate geometric gates in tripod systems. We have discussed how standard STA techniques designed for the transfer of a single known state are problematic due to STA-induced modification of dynamical and geometric phases. We have also shown a set of protocols that overcome this seeming limitation and discussed the advantages of using accelerated gates in the presence of dissipation and Hamiltonian uncertainties: the accelerated gate preserves the robustness against parameter variations and allows one to be fast enough to overcome thresholds set by relaxation and dephasing times. Our accelerated control scheme can be implemented in a variety of state-of-the-art qubit implementations.

Our work also suggests that accelerated geometric-based two-qubit gates could be developed for a variety of systems. In particular, it could greatly benefit superconducting-based architectures where two-qubit gates are still the main limitation preventing the realization of high-fidelity complex gate sequences.

\section{ACKNOWLEDGMENT}

This work was supported by the Army Research Office under Grant No. W911NF-19-1-0328.

\section{APPENDIX A: AVERAGE GATE FIDELITY WITH UNITARY EVOLUTION}

In this Appendix we show how to use a Magnus expansion to obtain approximate solutions of the Schrödinger equation

$$
i \partial_{t} \hat{U}(t)=\hat{H}(t) \hat{U}(t),
$$

where $\hat{H}(t)$ is defined in Eq. (5) of the text. We focus on the special case where the control field phase $\gamma(t)$ is given by Eq. (16). The special form of $\gamma(t)$ and the symmetry of the function $\theta(t)$ [see Eq. (8)] allow us to split the evolution into two distinct STIRAP processes defined by the Hamiltonians

$$
\hat{H}_{1}(t)=\frac{\Omega_{0}}{2}\left[\sin \left[\theta_{1}(t)\right]|\tilde{1}\rangle\left\langle e\left|+\cos \left[\theta_{1}(t)\right]\right| a\right\rangle\langle e|+\text { H.c. }\right],
$$

which describes the first half of the evolution that brings the system from $|\tilde{1}\rangle$ to $|a\rangle$, and

$$
\hat{H}_{2}(t)=\frac{\Omega_{0}}{2}\left[\cos \left[\theta_{1}(t)\right]|\tilde{1}\rangle\left\langle e\left|+e^{i \gamma_{0}} \sin \left[\theta_{1}(t)\right]\right| a\right\rangle\langle e|+\text { H.c. }\right],
$$

which describes the second half of the evolution that brings $|a\rangle$ back to $|\tilde{1}\rangle$. Here, $\theta_{1}(t)=\pi P(t) / 2$ [see Eq. (9)] is defined for $t \in\left[0, t_{g} / 2\right]$ and we have used the symmetry of the function $\theta(t)$ [see Eq. (8)] to obtain Eq. (A3). Within this framework the evolution operator $\hat{U}$ can be parametrized as

$$
\hat{U}(t)= \begin{cases}\hat{U}_{1}(t), & 0 \leqslant t<\frac{t_{g}}{2}, \\ \hat{U}_{2}\left(t-t_{g} / 2\right) \hat{U}_{1}\left(t_{g} / 2\right), & \frac{t_{g}}{2} \leqslant t<t_{g},\end{cases}
$$

where $\hat{U}_{1}(t)$ is generated by $\hat{H}_{1}(t)$ [see Eq. (A2)] and $\hat{U}_{2}(t)$ is generated by $\hat{H}_{2}(t)$ [see Eq. (A3)]. We stress that $\hat{U}(t)$ is continuous at $t=t_{g} / 2$ because the actual coupling strength between $|a\rangle$ and $|e\rangle$ is 0 , which allows us in the first place to have a phase jump.

It is useful to look for solutions of Eq. (A1) in the adiabatic frame. We can transform Eqs. (A2) and (A3) to the adiabatic frame by using the frame-change operator $\hat{S}_{\text {ad }}(t)$ defined in Eq. (10) of the text with

$\left|d_{2}(t)\right\rangle=\left\{\begin{array}{lll}\cos \left[\theta_{1}(t)\right]|\tilde{1}\rangle-\sin \left[\theta_{1}(t)\right]|a\rangle & \text { for } & \hat{H}_{1}(t), \\ \sin \left[\theta_{1}(t)\right]|\tilde{1}\rangle-e^{i \gamma_{0}} \cos \left[\theta_{1}(t)\right]|a\rangle & \text { for } & \hat{H}_{2}(t),\end{array}\right.$

and

$$
\left|b_{ \pm}(t)\right\rangle=\left\{\begin{array}{l}
\frac{1}{\sqrt{2}}\left( \pm \sin \left[\theta_{1}(t)\right]|\tilde{1}\rangle \pm \cos \left[\theta_{1}(t)\right]|a\rangle+|e\rangle\right) \\
\text { for } \quad \hat{H}_{1}(t), \\
\frac{1}{\sqrt{2}}\left( \pm \cos \left[\theta_{1}(t)\right]|\tilde{1}\rangle \pm e^{i \gamma_{0}} \sin \left[\theta_{1}(t)\right]|a\rangle+|e\rangle\right) \\
\text { for } \quad \hat{H}_{2}(t) .
\end{array}\right.
$$

We find

$$
\hat{H}_{\mathrm{ad}, 1(2)}(t)=-\frac{\Omega_{0}}{2} \hat{J}_{z, \text { ad }} \pm \dot{\theta}_{1}(t) \hat{J}_{y, \text { ad }},
$$

where $\hat{H}_{\mathrm{ad}, 1}\left[\hat{H}_{\mathrm{ad}, 2}\right]$ denotes $\hat{H}_{1}(t)\left[\hat{H}_{2}(t)\right]$ in the adiabatic frame. We have introduced the spin operators $\hat{J}_{z \text {,ad }}=$ $\left(\left|b_{-}\right\rangle\left\langle b_{-}|-| b_{+}\right\rangle\left\langle b_{+}\right|\right)$and $\hat{J}_{y, \text { ad }}=\left(i\left|d_{2}\right\rangle\left\langle b_{+}|-i| d_{2}\right\rangle\left\langle b_{-}\right|+\right.$ H.c. $) / \sqrt{2}$. We note that the remaining spin operator $\hat{J}_{x, \text { ad }}$ is introduced in the text following Eq. (32). It is convenient to transform Eq. (A7) to the interaction picture generated by $\hat{H}_{0, \text { ad }}=-\Omega_{0} \hat{J}_{z \text {,ad }} / 2$ to perform the Magnus expansion; this yields

$$
\begin{aligned}
\hat{V}_{I, 1(2)}(t)= & \mp i e^{-i \frac{\Omega_{0}}{2} t} \frac{\dot{\theta}_{1}(t)}{\sqrt{2}}\left|b_{-}\right\rangle\left\langle d_{2}\left| \pm i e^{i \frac{\Omega_{0}}{2} t} \frac{\dot{\theta}_{1}(t)}{\sqrt{2}}\right| b_{+}\right\rangle\left\langle d_{2}\right| \\
& + \text { H.c. }
\end{aligned}
$$


It is useful to note that the dynamics generated by $\hat{V}_{I, 1(2)}(t)$ can be parametrized as

$$
\begin{aligned}
\hat{U}_{I, 1(2)}(t)= & \exp \left[-i\left(\Delta(t) \hat{J}_{z, \text { ad }} \pm \Omega_{x}(t) \hat{J}_{x, \text { ad }} \pm \Omega_{y}(t) \hat{J}_{y, \text { ad }}\right)\right] \\
= & \exp \left[-i \xi(t)\left(n_{z}(t) \hat{J}_{z, \text { ad }} \pm n_{x}(t)\right.\right. \\
& \left.\left.\times \hat{J}_{x, \text { ad }} \pm n_{y}(t) \hat{J}_{y, \text { ad }}\right)\right]
\end{aligned}
$$

with $\xi(t)=\sqrt{\Delta^{2}(t)+\Omega_{x}^{2}(t)+\Omega_{y}^{2}(t)}, \quad n_{x}(t)=\Omega_{x}(t) / \xi(t)$, $n_{y}(t)=\Omega_{y}(t) / \xi(t)$, and $n_{z}(t)=\Delta(t) / \xi(t)$. This form allows us to get an exact representation for $\hat{U}_{I, 1(2)}(t)$ by expanding the exponential into a series and using the properties of the spin operators. The functions $\Delta(t), \Omega_{x}(t)$, and $\Omega_{y}(t)$ are found perturbatively using a fourth-order Magnus expansion [35]. We find that at $t=t_{g} / 2$ these functions evaluate to

$$
\begin{aligned}
\Delta\left(t_{g} / 2\right) & =\frac{a_{1}}{\Omega_{0} t_{g}}+\frac{a_{2}}{\left(\Omega_{0} t_{g}\right)^{3}}, \\
\Omega_{x}\left(t_{g} / 2\right) & =b_{1} \frac{\sin ^{2}\left(\frac{\Omega_{0} t_{g}}{8}\right)}{\left(\Omega_{0} t_{g}\right)^{3}}+b_{2} \frac{\sin \left(\frac{\Omega_{0} t_{g}}{4}\right)}{\left(\Omega_{0} t_{g}\right)^{4}}, \\
\Omega_{y}\left(t_{g} / 2\right) & =c_{1} \frac{\sin \left(\frac{\Omega_{0} t_{g}}{4}\right)}{\left(\Omega_{0} t_{g}\right)^{3}}+c_{2} \frac{\cos ^{2}\left(\frac{\Omega_{0} t_{g}}{8}\right)}{\left(\Omega_{0} t_{g}\right)^{4}},
\end{aligned}
$$

with $a_{1}=-5 \pi^{2} / 7, \quad a_{2}=4500 \pi^{4} / 2431-960 \pi^{2} / 7, \quad b_{1}=$ $3840 \pi, b_{2}=960 \pi\left(336+5 \pi^{2}\right) / 7, c_{1}=-1920 \pi$, and $c_{2}=$ $-1920 \pi\left(336+5 \pi^{2}\right) / 7$. Finally, the evolution operators are given by

$$
\hat{U}_{1(2)}(t)=\hat{S}_{\mathrm{ad}, 1(2)} \hat{U}_{0}(t) \hat{U}_{I, 1(2)}(t) \hat{S}_{\mathrm{ad}, 1(2)}^{\dagger}(0),
$$

with $\hat{U}_{0}(t)=\exp \left[i \Omega_{0} t \hat{S}_{z, \text { ad }} / 2\right]$.

We can evaluate Eq. (22) with $\hat{O}=\hat{O}_{q}$ [see text following Eq. (23)] and $\hat{U}_{G}=\hat{U}\left(t_{g}\right)$ [see Eq. (A4)] (not shown due to the length of the result). To get Eq. (24), one further needs to expand the trigonometric functions involved in the result to fourth order in $\xi(t)$ and collect terms up to sixth order in $1 /\left(\Omega_{0} t_{g}\right)$.

\section{APPENDIX B: AVERAGE MAP FIDELITY WITH EXCITED-STATE DEPHASING}

In this section we present the general framework allowing us to evaluate perturbatively the average fidelity of the qubit map [cf. Eq. (46)] for the accelerated gate in the presence of excited-state dephasing. We start by defining the modified Hamiltonian with the SATD correction

$$
\hat{H}_{\text {mod }}(t)=\hat{H}(t)+\hat{W}_{\mathrm{SATD}}(t),
$$

where $\hat{H}(t)$ is the Hamiltonian of the tripod system written in terms of the new qubit states [cf. Eq. (5)] and

$$
\begin{aligned}
\hat{W}_{\mathrm{SATD}}= & \frac{2 \Omega_{0} \ddot{\theta}(t)}{\Omega_{0}^{2}+4 \dot{\theta}^{2}(t)}[\cos [\theta(t)]|\tilde{1}\rangle\langle e| \\
& \left.-e^{i \gamma(t)} \sin [\theta(t)]|a\rangle\langle e|+\text { H.c. }\right] .
\end{aligned}
$$

In the frame defined by the SATD dressing [see Eqs. (32) and (41)], the master equation describing the evolution of the tripod system with excited dephasing is given by

$$
\begin{aligned}
\partial_{t} \hat{\rho}_{\mathrm{dr}}(t)= & -i\left[\hat{H}_{\mathrm{mod}, \mathrm{dr}}(t), \hat{\rho}_{\mathrm{dr}}(t)\right]+\Gamma_{\varphi, e} \sum_{\substack{i, j, k, l=\\
\tilde{b}_{-}, \tilde{b}_{+}, \tilde{d}_{2}}} c_{i}(t) \\
& \times c_{j}^{*}(t)|i\rangle\left\langle j\left|\hat{\rho}_{\mathrm{dr}}(t) c_{k}(t) c_{l}^{*}(t)\right| k\right\rangle\langle l| \\
& -\frac{1}{2} \Gamma_{\varphi, e} \sum_{\substack{i, j=\tilde{c}_{i} \\
\tilde{b}, \tilde{b}_{+}, \tilde{d}_{2}}}\left\{c_{i}(t) c_{j}^{*}(t)|i\rangle\langle j|, \hat{\rho}_{\mathrm{dr}}(t)\right\}_{+},
\end{aligned}
$$

with $c_{\tilde{b}_{-}}(t)=c_{\tilde{b}_{+}}(t)=1 /\left(\sqrt{2} \sqrt{1+4 \dot{\theta}^{2}(t) / \Omega_{0}^{2}}\right)$ and $c_{\tilde{d}_{2}}=$ $2 i \dot{\theta}(t) /\left(\Omega_{0} \sqrt{1+4 \dot{\theta}^{2}(t) / \Omega_{0}^{2}}\right)$.

Using a superoperator formalism Eq. (B3) can be written as

$$
\partial_{t} \hat{\rho}_{\mathrm{dr}}(t)=\left[\boldsymbol{\ell}_{0}(t)+\boldsymbol{\ell}_{\varphi}(t)\right] \hat{\rho}_{\mathrm{dr}},
$$

where $\boldsymbol{\ell}_{0}(t)=i\left[\hat{H}_{\text {mod,dr }}^{\top}(t) \otimes \mathbb{1}-\mathbb{1} \otimes \hat{H}_{\text {mod,dr }}(t)\right]$ and $\boldsymbol{\ell}_{\varphi}=$ $\hat{L}^{*}(t) \otimes \hat{L}(t)-(1 / 2) \mathbb{1} \otimes \hat{L}^{\top}(t) \hat{L}(t)-(1 / 2) \hat{L}^{\top}(t) \hat{L}^{*}(t) \otimes \mathbb{1}$ and we have defined $\hat{L}_{\varphi}(t)=\sqrt{\Gamma_{\varphi, e}} \sum_{i, j} c_{i}(t) c_{j}^{*}(t)|i\rangle\langle j|$ with $i, j \in\left\{\tilde{b}_{-}, \tilde{b}_{+}, \tilde{d}_{2}\right\}$. We have also introduced the complex conjugation and transpose operations, denoted $\hat{L}^{*}(t)$ and $\hat{L}^{\top}(t)$, respectively.

To find approximate solutions of Eq. (B4) it is convenient to work in the interaction picture defined by $\boldsymbol{\ell}_{0}(t)$. In this frame Eq. (B4) reduces to

$$
\partial_{t} \hat{\rho}_{\mathrm{dr}, \mathrm{I}}(t)=\boldsymbol{\ell}_{\varphi, I}(t) \hat{\rho}_{\mathrm{dr}, \mathrm{I}}(t),
$$

where $\boldsymbol{\ell}_{\varphi, I}(t)=\mathcal{L}_{0}^{\dagger}(t) \boldsymbol{\ell}_{\varphi}(t) \mathcal{L}_{0}(t)$ and $\mathcal{L}_{0}(t)$ is the solution of $\partial_{t} \hat{\rho}_{\mathrm{dr}}(t)=\boldsymbol{\ell}_{0}(t) \hat{\rho}_{\mathrm{dr}}(t)$, i.e., $\hat{\rho}_{\mathrm{dr}}(t)=\mathcal{L}_{0}(t) \hat{\rho}_{\mathrm{dr}}(0)$. Within this framework the solution of Eq. (B4) is given by $\hat{\rho}_{\mathrm{dr}}(t)=$ $\mathcal{L}_{0}(t) \mathcal{L}_{I}(t) \hat{\rho}_{\mathrm{dr}}(0)$, where $\mathcal{L}_{I}(t)$ is a solution of Eq. (B5). Using a first-order Magnus expansion [35], we can approximate $\mathcal{L}_{I}(t)$ by

$$
\mathcal{L}_{I}(t) \simeq \exp \left[\int_{0}^{t} d t_{1} \boldsymbol{\ell}_{\varphi, I}\left(t_{1}\right)\right] \simeq \mathbb{1}+\int_{0}^{t} d t_{1} \boldsymbol{\ell}_{\varphi, I}\left(t_{1}\right) .
$$

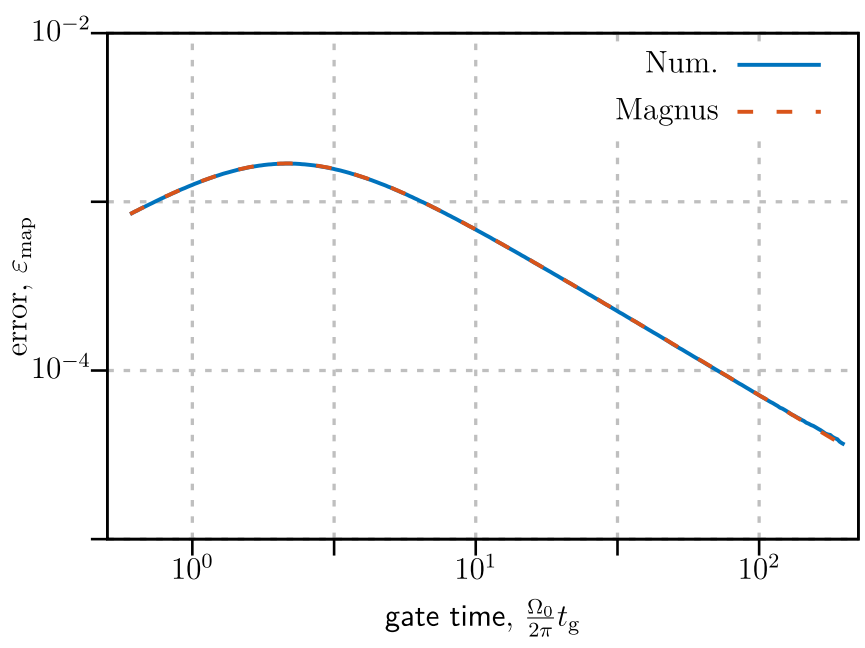

FIG. 6. Comparison of the average error $\varepsilon_{\text {map }}=1-\bar{F}_{\text {map }}$ calculated numerically (solid blue trace) vs using Eq. (49) (dashed orange trace). We used $\alpha=\pi / 4, \beta=0, \gamma_{0}=\pi, \Gamma_{\varphi, e}=\Omega_{0} /(2 \pi \times 100)$. 
This leads to an approximate solution for $\hat{\rho}_{\mathrm{dr}}(t)$,

$$
\hat{\rho}_{\mathrm{dr}}(t) \simeq \mathcal{L}_{0}(t)\left[\mathbb{1}+\int_{0}^{t} d t_{1} \boldsymbol{\ell}_{\varphi, I}\left(t_{1}\right)\right]
$$

which can be used to evaluate Eq. (46).
In Fig. 6 we plot the error $\varepsilon_{\text {map }}$ as a function of the gate time for $\alpha=\pi / 4, \beta=0, \gamma_{0}=\pi$, and $\Gamma_{\varphi, e}=\Omega_{0} /(2 \pi \times 100)$ for the accelerated protocol calculated numerically (solid blue trace) and using Eq. (49) (dashed orange trace). As stated in the text, the approximate analytical result is in very good agreement with the numerical results.
[1] L.-M. Duan, J. I. Cirac, and P. Zoller, Geometric manipulation of trapped ions for quantum computation, Science 292, 1695 (2001).

[2] Z. Kis and F. Renzoni, Qubit rotation by stimulated raman adiabatic passage, Phys. Rev. A 65, 032318 (2002).

[3] D. Møller, L. B. Madsen, and K. Mølmer, Geometric phases in open tripod systems, Phys. Rev. A 77, 022306 (2008).

[4] L. Mandelstam and I. Tamm, The uncertainty relation between energy and time in non-relativistic quantum mechanics, in Selected Papers, edited by B. M. Bolotovskii, V. Y. Frenkel, and R. Peierls (Springer, Berlin, 1991), pp. 115-123.

[5] N. Margolus and L. B. Levitin, The maximum speed of dynamical evolution, Physica D: Nonlin. Phenom. 120, 188 (1998).

[6] S. Deffner and E. Lutz, Quantum Speed Limit for NonMarkovian Dynamics, Phys. Rev. Lett. 111, 010402 (2013).

[7] A. C. Santos and M. S. Sarandy, Superadiabatic controlled evolutions and universal quantum computation, Sci. Rep. 5, 15775 (2015).

[8] D. P. Pires, M. Cianciaruso, L. C. Céleri, G. Adesso, and D. O. Soares-Pinto, Generalized Geometric Quantum Speed Limits, Phys. Rev. X 6, 021031 (2016).

[9] I. Marvian, R. W. Spekkens, and P. Zanardi, Quantum speed limits, coherence, and asymmetry, Phys. Rev. A 93, 052331 (2016).

[10] M. Demirplak and S. A. Rice, Adiabatic population transfer with control fields, J. Phys. Chem. A 107, 9937 (2003).

[11] M. Demirplak and S. A. Rice, Assisted adiabatic passage revisited, J. Phys. Chem. B 109, 6838 (2005).

[12] M. V. Berry, Transitionless quantum driving, J. Phys. A: Math. Theor. 42, 365303 (2009).

[13] S. Ibáñez, X. Chen, E. Torrontegui, J. G. Muga, and A. Ruschhaupt, Multiple Schrödinger Pictures and Dynamics in Shortcuts to Adiabaticity, Phys. Rev. Lett. 109, 100403 (2012).

[14] M. G. Bason, M. Viteau, N. Malossi, P. Huillery, E. Arimondo, D. Ciampini, R. Fazio, V. Giovannetti, R. Mannella, and O. Morsch, High-fidelity quantum driving, Nat. Phys. 8, 147 (2011).

[15] J.-F. Schaff, X.-L. Song, P. Capuzzi, P. Vignolo, and G. Labeyrie, Shortcut to adiabaticity for an interacting Bose-Einstein condensate, EPL (Europhys. Lett.) 93, 23001 (2011).

[16] J. Zhang, J. H. Shim, I. Niemeyer, T. Taniguchi, T. Teraji, H. Abe, S. Onoda, T. Yamamoto, T. Ohshima, J. Isoya, and D. Suter, Experimental Implementation of Assisted Quantum Adiabatic Passage in a Single Spin, Phys. Rev. Lett. 110, 240501 (2013).

[17] S. An, D. Lv, A. del Campo, and K. Kim, Shortcuts to adiabaticity by counterdiabatic driving for trapped-ion displacement in phase space, Nat. Commun. 7, 12999 (2016).
[18] Y.-X. Du, Z.-T. Liang, Y.-C. Li, X.-X. Yue, Q.-X. Lv, W. Huang, X. Chen, H. Yan, and S.-L. Zhu, Experimental realization of stimulated raman shortcut-to-adiabatic passage with cold atoms, Nat. Commun. 7, 12479 (2016).

[19] B. B. Zhou, A. Baksic, H. Ribeiro, C. G. Yale, F. J. Heremans, P. C. Jerger, A. Auer, G. Burkard, A. A. Clerk, and D. D. Awschalom, Accelerated quantum control using superadiabatic dynamics in a solid-state lambda system, Nat. Phys. 13, 330 (2016).

[20] J. Kölbl, A. Barfuss, M. S. Kasperczyk, L. Thiel, A. A. Clerk, H. Ribeiro, and P. Maletinsky, Initialization of Single Spin Dressed States Using Shortcuts to Adiabaticity, Phys. Rev. Lett. 122, 090502 (2019).

[21] R. Srinivas, S. C. Burd, R. T. Sutherland, A. C. Wilson, D. J. Wineland, D. Leibfried, D. T. C. Allcock, and D. H. Slichter, Trapped-Ion Spin-Motion Coupling with Microwaves and a Near-Motional Oscillating Magnetic Field Gradient, Phys. Rev. Lett. 122, 163201 (2019).

[22] V. E. Manucharyan, J. Koch, L. I. Glazman, and M. Devoret, Fluxonium: Single Cooper-pair circuit free of charge offsets, Science 326, 113 (2009).

[23] N. Earnest, S. Chakram, Y. Lu, N. Irons, R. K. Naik, N. Leung, L. Ocola, D. A. Czaplewski, B. Baker, J. Lawrence, J. Koch, and D. I. Schuster, Realization of a $\Lambda$ System with Metastable States of a Capacitively Shunted Fluxonium, Phys. Rev. Lett. 120, 150504 (2018).

[24] A. Baksic, H. Ribeiro, and A. A. Clerk, Speeding Up Adiabatic Quantum State Transfer by Using Dressed States, Phys. Rev. Lett. 116, 230503 (2016).

[25] E. Sjöqvist, D. M. Tong, L. M. Andersson, B. Hessmo, M. Johansson, and K. Singh, Non-adiabatic holonomic quantum computation, New J. Phys. 14, 103035 (2012).

[26] E. Sjöqvist, Nonadiabatic holonomic single-qubit gates in offresonant $\Lambda$ systems, Phys. Lett. A 380, 65 (2016).

[27] A. A. Abdumalikov, Jr., J. M. Fink, K. Juliusson, M. Pechal, S. Berger, A. Wallraff, and S. Filipp, Experimental realization of non-Abelian non-adiabatic geometric gates, Nature 496, 482 (2013)

[28] B. B. Zhou, P. C. Jerger, V. O. Shkolnikov, F. J. Heremans, G. Burkard, and D. D. Awschalom, Holonomic Quantum Control by Coherent Optical Excitation in Diamond, Phys. Rev. Lett. 119, 140503 (2017).

[29] T. Yan, B.-J. Liu, K. Xu, C. Song, S. Liu, Z. Zhang, H. Deng, Z. Yan, H. Rong, K. Huang, M.-H. Yung, Y. Chen, and D. Yu, Experimental Realization of Nonadiabatic Shortcut to Non-Abelian Geometric Gates, Phys. Rev. Lett. 122, 080501 (2019).

[30] B.-J. Liu, Z.-H. Huang, Z.-Y. Xue, and X.-D. Zhang, Superadiabatic holonomic quantum computation in cavity QED, Phys. Rev. A 95, 062308 (2017). 
[31] K. Bergmann, H. Theuer, and B. W. Shore, Coherent population transfer among quantum states of atoms and molecules, Rev. Mod. Phys. 70, 1003 (1998).

[32] N. V. Vitanov, A. A. Rangelov, B. W. Shore, and K. Bergmann, Stimulated Raman adiabatic passage in physics, chemistry, and beyond, Rev. Mod. Phys. 89, 015006 (2017).

[33] S. Pancharatnam, Generalized theory of interference, and its applications, Proc. Indian Acad. Sci. Sec. A 44, 247 (1956).

[34] M. V. Berry, Quantal phase factors accompanying adiabatic changes, Proc. R. Soc. London Ser. A 392, 45 (1984).

[35] W. Magnus, On the exponential solution of differential equations for a linear operator, Commun. Pure Applied. Math. 7, 649 (1954).

[36] D. A. Lidar, A. T. Rezakhani, and A. Hamma, Adiabatic approximation with exponential accuracy for many-body systems and quantum computation, J. Math. Phys. 50, 102106 (2009).

[37] A. T. Rezakhani, A. K. Pimachev, and D. A. Lidar, Accuracy versus run time in an adiabatic quantum search, Phys. Rev. A 82, 052305 (2010).

[38] N. Wiebe and N. S. Babcock, Improved error-scaling for adiabatic quantum evolutions, New J. Phys. 14, 013024 (2012).

[39] L. H. Pedersen, N. M. Møller, and K. Mølmer, Fidelity of quantum operations, Phys. Lett. A 367, 47 (2007).

[40] M. Demirplak and S. A. Rice, On the consistency, extremal, and global properties of counterdiabatic fields, J. Chem. Phys. 129, 154111 (2008).

[41] M. D. Bowdrey, D. K. Oi, A. Short, K. Banaszek, and J. Jones, Fidelity of single qubit maps, Phys. Lett. A 294, 258 (2002). 